\title{
Evaluating the Efficacy and Trend of Sinus Surgery
}

\author{
Masa Petrovic ${ }^{1}$ Arash Shamsian ${ }^{1} \quad$ Martin L. Hopp ${ }^{1} \quad$ Narine Vardanyan $^{1}$ \\ ${ }^{1}$ Department of Otolaryngology, Cedars-Sinai Sinus Center, Los \\ Angeles, California, United States \\ Address for correspondence Masa Petrovic, B.S., Department of \\ Otolaryngology, Cedars-Sinai Sinus Center, 8631 W 3rd Street \#440E, Los \\ Angeles, CA 90048, United States (e-mail: 5rovicmasa@gmail.com).
} Int Arch Otorhinolaryngol 2020;24(4):e407-e412.

\begin{abstract}
Keywords

- quality of life

- sinusitis

- paranasal sinuses

Introduction From April 2009 to December 2016, 661 consecutive patients undergoing sinus surgery completed a quality of life (QOL) questionnaire (SNOT-22) preoperatively and at 3,6, and 12 months postoperatively.

Objective (1) To evaluate the long-term efficacy of sinus surgery using QOL instruments. (2) To determine the optimal evaluation time for surgical efficacy. (3) To determine if surgical results improve with yearly experience.

Methods The prospective study patients were split into two groups: Group A, those who completed the initial preoperative evaluation and all postoperative evaluations, and Group B, who completed the preoperative questionnaire and at least one but not all of the postoperative questionnaires. Group A included 93 patients. Group B included 240 patients at 3 months, 180 at 6 months, and 121 at 12 months postoperatively.

Results Group A efficacy reported at 3 months was $82.8 \%, 80.6 \%$ at 6 months, and $84.9 \%$ at 12 months postoperatively. Group B efficacy reported at 3 months was $71.3 \%$, $78.3 \%$ at 6 months, and $84.3 \%$ at 12 months postoperatively. An 8-year trend analysis of year-to-year 12 months postoperative data illustrates a significant improvement with an analysis of variance (ANOVA) linear rate of $1.594(p \leq 0.12)$.

Conclusion The 8-year trend at 12 months postoperatively shows a positive improvement in surgical results. Patients undergoing sinus surgery at tertiary medical center showed $84.9 \%$ improvement in sinus disease symptoms by 12 months postoperatively. Long-term improvement analysis showed no difference between 6 months postoperatively and 12 months, signifying 6 months as an effective evaluation for surgical efficacy.
\end{abstract}

\section{Introduction}

Chronic rhinosinusitis (CRS) is characterized by inflammation of the paranasal sinuses persistent for 12 weeks or longer despite treatment, impacting both physical and mental health. Chronic rhinosinusitis is a prevalent condition affecting up to $16 \%$ of Americans aged between 18 and 59 years old, with $6.8 \%$ of Americans after the age of 69 years old. ${ }^{1}$ Chronic rhinosinusitis has been found to be highly correlated to a diminished quality of life (QOL) and is considered a burden on healthcare.

Chronic rhinosinusitis is considered to be an underresearched chronic disease when compared with other dis- eases that have a similar burden in regards to healthcare, prevalence, and $\mathrm{QOL}^{2}{ }^{2}$ The economic burden of CRS is increasing at an upward trend with the most recent estimate in 2015 showing total yearly medical expenditures for CRS in the United States to be between 60.6 billion and 64.9 billion US dollars per year (treatment for CRS ranges from 5,560 USD to 5,955 USD per person). ${ }^{3}$ Aside from the costs of healthcare, there are indirect costs associated with CRS. Chronic rhinosinusitis has been placed among the top 10 debilitating medical conditions placed among angina, congestive heart failure (CHF), chronic obstructive pulmonary disease (COPD), and chronic back pain, as well as one of the top conditions affecting employers similar to disease states such as acute received

February 17, 2019

accepted

October 20, 2019
DOI https://doi.org/

10.1055/s-0039-3402436. ISSN 1809-9777.
Copyright $\odot 2020$ by Thieme Revinter

Publicações Ltda, Rio de Janeiro, Brazil
License terms

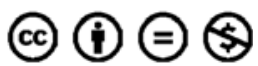


myocardial infarction, COPD, and trauma to the spine and spinal cord. ${ }^{4,5}$

Patients with CRS reported similar QOL in patients with other chronic conditions such as cancer, arthritis, asthma, and inflammatory bowel disease. ${ }^{6}$ Furthermore, patients with CRS are reported to have much lower bodily pain scores and social functioning scores than those with CHF, COPD, angina, and back pain. 7

The Sino-Nasal Outcome Test (SNOT-22) is an evidencebased, verified, symptom-specific QOL measurement to evaluate the severity of sinus disease, consisting of 22 questions in which patients are asked to evaluate their symptoms on a scale of 0 to 5, 0 indicating no problem, and 5 indicating the symptom to be as severe as it can be. According to the research by Rudmik et al on the predictive value of the preoperative SNOT-22 score, a score change of 9 points or greater is considered as a clinically significant change. ${ }^{8}$ The SNOT-22 is meant to illustrate changes between the pre-and postoperative. At the preoperative, patients are asked to indicate the symptoms they consider to be most important to them. In contrast, postoperative SNOT-22 scores evaluate the surgical efficacy through symptoms after surgery with the yearly QOL standards set as monthly follow-ups. By using SNOT-22, we can determine the long-term efficacy of sinus surgery, the optimal evaluation time for surgical efficacy, and if surgical results improve with yearly experience.

\section{Methods}

The present study was approved by the Office of Research Compliance and Quality Improvement of the Medical Center (Pro00039371).

The patient sample is the hospital population of all CRS patients subdivided by severity of disease according to the SNOT-22 category. Part of the standard of care at a tertiary medical center, patients $\geq 18$ years old undergoing sinus surgery are given a SNOT-22 form to complete preoperatively. Chronic rhinosinusitis was diagnosed with a minimum of 12 weeks of symptomatic sinusitis episodes, unresponsiveness to antibiotics and steroids in any form, and positive CT scan findings. All of the patients underwent transnasal endoscopic sinus surgery, including septoplasty and turbinoplasty. The SNOT-22 QOL instrument provided substantial insight for a prospective study. Quality of life measurements are of overall hospital surgical morbidity of patients diagnosed with CRS undergoing sinus surgery and, therefore, would not include any subcategories such as nasal polyps. At 3 months, 6 months, and 12 months postoperatively, patients were asked to complete another SNOT-22 form. Data was collected by a project coordinator via Health Insurance Portability and Accountability Act (HIPAA) compliant measures: mail/email/telephone. At the time of data analysis, all patient identifying factors were concealed.

The population sample from April 2009 to December 2016 consists of 661 consecutive patients, 14 of which were indicated to be revision surgeries. All of the patients were asked to complete the SNOT-22 preoperatively, and at 3, 6, and 12 months postoperatively.
In the prospective study, patients were split into two groups: Group A, those who completed the initial preoperative evaluation and all postoperative evaluations, and Group B who completed at least one but not all of the postoperative questionnaires. In addition, patients who had a preoperative SNOT-22 score $<9$ were excluded, since no clinically significant change can be determined. For Group A, only data from between April 2009 and April 2016 was used. Group A consisted of 93 patients who completed all 4 milestone SNOT-22 forms with 553 patients lost to follow-up. Alternatively, Group B consisted of 240 patients at 3 months, 180 at 6 months, and 121 at 12 months. The remainder of the patients was lost to follow-up.

For Group B, data from April 2009 to December 2016 was used. Group B was created to observe a much larger population size, since it focused on all the data available for each of the milestones ( 3 months, 6 months, and 12 months postoperatively) compared with the initial score. Therefore, Group B also consisted of patients that may have only reached the 3 months postoperative milestone, who would have been excluded from Group A at the time of the most recent data analysis.

\section{Results}

From April 2009 to April 2016, 646 patients completed SNOT-22 questionnaires with the initial preoperative evaluation and all postoperative evaluations. From that population sample, 93 patients met the standard to be included in Group A. From the 8-year review of patients, 553 patients (85.6\%) had been lost to follow-up and, as a result, were excluded from Group A. According to the study by Hopkins et $\mathrm{al}^{9}{ }^{9} \mathrm{a}$ norm of $\sim 35 \%$ loss to follow-up is expected.

Group A results are broken down in - Table 1. Group A showed overall surgical success rates of $82.8 \%$ at 3 months, $80.6 \%$ at 6 months, and $84.9 \%$ at 12 months postoperatively ( $p$ $\leq 0.01$ ), with an average SNOT-22 score change of 24.4 at 12 months from their initial score (-Fig. 1). An 8-year trend analysis of year-to-year 12 months postoperative data illustrated significant improvement over time with an analysis of variance (ANOVA) linear rate of $1.594(p \leq 0.12)$, as seen in -Fig. 2. For each postoperative period (3 months, 6 months, and 12 months), a significant increase is seen in the trend line.

In 2009, 14 patients in Group A had a preoperative mean score of 43.3. At 3 months postoperatively, the efficacy was reported at $78.6 \%$ improvement, $7.1 \%$ the same, and $14.3 \%$ worsened, with a mean SNOT-22 score change (MSSC) of 19.2. At 6 months, the rates were reported at $85.7 \%$ improvement, $7.1 \%$ the same, and $7.1 \%$ worsened, and with an MSSC of 25.0 from their preoperative score. At 12 months, the MSSC was at 20.2 and rates were reported as they were at 3 months (78.6\% improvement, $7.1 \%$ the same, and $14.3 \%$ worsened).

In 2010, 19 patients in Group A had a preoperative mean score of 42.1. At 3 months postoperatively, the efficacy was reported at $84.2 \%$ improvement, $15.8 \%$ the same, and $0 \%$ worsened from their preoperative SNOT-22 score, with an 


\begin{tabular}{|c|c|c|c|c|c|c|c|c|c|c|}
\hline & $\frac{\stackrel{0}{\Xi}}{\frac{\pi}{2}}$ & $\begin{array}{l}\overline{0} \\
\dot{0} \\
\mathrm{~V} I\end{array}$ & $\begin{array}{l}\overline{0} \\
\dot{0} \\
\mathrm{~V} 1\end{array}$ & $\begin{array}{l}\overline{0} \\
\dot{\dot{v}} \\
\mathrm{~V} 1\end{array}$ & $\begin{array}{l}\bar{\delta} \\
\dot{0} \\
\dot{V}\end{array}$ & $\begin{array}{l}\overline{0} \\
\dot{\sigma} \\
\mathrm{V}\end{array}$ & $\mid \begin{array}{l}\bar{\delta} \\
\dot{v} \\
\mathrm{~V}\end{array}$ & $\begin{array}{l}\overline{0} \\
\dot{0} \\
\dot{v}\end{array}$ & $\begin{array}{l}\overline{0} \\
\dot{0} \\
\mathrm{~V}\end{array}$ & $\mid \begin{array}{l}\overline{0} \\
\dot{0} \\
\dot{v}\end{array}$ \\
\hline & 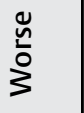 & 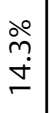 & $\begin{array}{c}\stackrel{\circ}{\mathrm{m}} \\
\text { மे }\end{array}$ & ㅇํํ & \begin{tabular}{|c}
$\stackrel{\circ}{0}$ \\
$\aleph_{\infty}$
\end{tabular} & 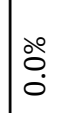 & 总 & $\begin{array}{l}\stackrel{\circ}{\circ} \\
0 \\
0\end{array}$ & $\begin{array}{l}\circ \\
0 \\
0\end{array}$ & ํํ \\
\hline 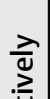 & 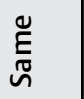 & $\begin{array}{l}\stackrel{\circ}{\check{.}} \\
\end{array}$ & $\begin{array}{l}\stackrel{\circ}{\circ} \\
\stackrel{0}{\circ}\end{array}$ & $\begin{array}{l}\stackrel{\circ}{0} \\
\dot{0}\end{array}$ & $\begin{array}{l}\text { Oें } \\
\text { ○े }\end{array}$ & $\begin{array}{l}\stackrel{\circ}{m} \\
\stackrel{+}{+}\end{array}$ & $\begin{array}{l}\stackrel{\circ}{m} \\
\stackrel{\sim}{\mp}\end{array}$ & 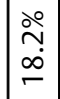 & $\begin{array}{l}\stackrel{\circ}{0} \\
\circ\end{array}$ & ○े \\
\hline 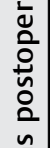 & 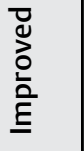 & $\mid \begin{array}{l}\grave{0} \\
\infty \\
i \\
\wedge\end{array}$ & $\begin{array}{l}\stackrel{\circ}{ } \\
\stackrel{亠}{\infty}\end{array}$ & $\begin{array}{l}\stackrel{0}{0} \\
\text { Lे } \\
\infty\end{array}$ & 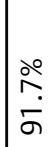 & $\begin{array}{l}\stackrel{\text { }}{\hat{N}} \\
\dot{\infty}\end{array}$ & $\begin{array}{l}\stackrel{2}{2} \\
\stackrel{2}{\infty} \\
\infty\end{array}$ & \begin{tabular}{|l|} 
○े \\
$\infty$ \\
$\dot{亠}$ \\
$\infty$
\end{tabular} & $\begin{array}{l}\text { 우 } \\
\dot{8} \\
0\end{array}$ & ㅇํ \\
\hline 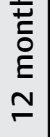 & 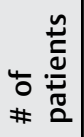 & \pm & $\stackrel{2}{\square}$ & $\stackrel{\sim}{\sim}$ & $\simeq$ & 1 & 1 & $\mp$ & $\stackrel{0}{\dot{m}}$ & n̆ \\
\hline & 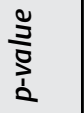 & $\begin{array}{l}\overline{0} \\
\dot{0} \\
\bar{v} \mid\end{array}$ & $\mid \begin{array}{c}\overline{0} \\
\dot{0} \\
\mathrm{~V} 1\end{array}$ & $\begin{array}{l}\bar{\delta} \\
\dot{\dot{v}} \\
\bar{v} \mid\end{array}$ & $\begin{array}{l}\overline{0} \\
\dot{0} \\
\mathrm{~V}\end{array}$ & $\begin{array}{l}\bar{\sigma} \\
\dot{0} \\
\mathrm{~V}\end{array}$ & $\mid \begin{array}{l}\bar{\delta} \\
\dot{v} \\
\mathrm{~V}\end{array}$ & $\begin{array}{l}\overline{0} \\
\dot{0} \\
\text { VV }\end{array}$ & $\begin{array}{l}\bar{\delta} \\
\dot{0} \\
\bar{V}\end{array}$ & $\begin{array}{l}\overline{0} \\
\dot{0} \\
\mathrm{~V}\end{array}$ \\
\hline & 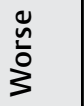 & 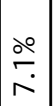 & $\mid \begin{array}{l}\circ \\
\circ \\
0 \\
0\end{array}$ & $\mid \begin{array}{l}\circ \\
0 \\
0\end{array}$ & \begin{tabular}{|l}
$\circ 0$ \\
0 \\
0
\end{tabular} & $\mid \begin{array}{l}\text { Oें } \\
\circ\end{array}$ & $\begin{array}{l}\stackrel{\circ}{\ominus} \\
\dot{\sim}\end{array}$ & $\begin{array}{l}\text { Oें } \\
0\end{array}$ & \begin{tabular}{|l}
$\stackrel{0}{0}$ \\
0 \\
0
\end{tabular} & సे \\
\hline$\overline{\mathrm{g}}$ & $\begin{array}{l}\underset{\tilde{E}}{N} \\
\text { N }\end{array}$ & 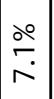 & $\mid \begin{array}{l}\stackrel{\circ}{\check{\sim}} \\
\dot{\sim}\end{array}$ & $\begin{array}{l}\stackrel{\circ}{0} \\
\dot{0} \\
\grave{1}\end{array}$ & $\begin{array}{l}\stackrel{\circ}{0} \\
\stackrel{\leftrightarrow}{\sim}\end{array}$ & $\begin{array}{l}\stackrel{\circ}{\tilde{m}} \\
\stackrel{+}{+}\end{array}$ & 吕 & 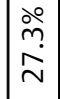 & 号 & 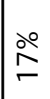 \\
\hline 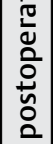 & 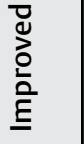 & $\begin{array}{l}\stackrel{2}{\hat{\imath}} \\
\dot{\omega} \\
\infty\end{array}$ & $\mid \begin{array}{l}\stackrel{\circ}{\sigma} \\
\infty \\
\triangleright\end{array}$ & $\mid \begin{array}{l}\stackrel{0}{0} \\
0 \\
\dot{0}\end{array}$ & $\begin{array}{l}\text { ○े } \\
\text { 수 }\end{array}$ & $\begin{array}{l}\stackrel{0}{1} \\
\hat{1} \\
\infty\end{array}$ & $\begin{array}{l}\stackrel{\circ}{\wedge} \\
\stackrel{1}{\infty} \\
\infty\end{array}$ & $\begin{array}{l}\stackrel{0}{\hat{N}} \\
\stackrel{N}{N}\end{array}$ & $\mid$ & ळे \\
\hline 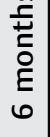 & 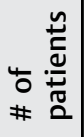 & 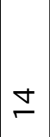 & 9 & $\stackrel{\sim}{\sim}$ & $\simeq$ & $r$ & 1 & $\mp$ & $\stackrel{0}{m}$ & n̆ \\
\hline & $\frac{\text { ŏ }}{\grave{c}}$ & $\begin{array}{l}\overline{0} \\
\dot{d} \\
\bar{v}\end{array}$ & $\left|\begin{array}{c}\overline{0} \\
\dot{d} \\
\bar{v}\end{array}\right|$ & $\left|\begin{array}{l}\overline{0} \\
\dot{d} \\
\dot{v}\end{array}\right|$ & $\mid \begin{array}{l}\overline{0} \\
\dot{0} \\
\bar{V}\end{array}$ & $\begin{array}{l}\overline{0} \\
\dot{0} \\
\mathrm{~V} \mid\end{array}$ & $\mid \begin{array}{l}\tilde{0} \\
\dot{0} \\
\dot{v}\end{array}$ & $\begin{array}{l}\overline{0} \\
\dot{0} \\
\bar{v} \mid\end{array}$ & $\mid \begin{array}{l}\overline{0} \\
\dot{0} \\
\bar{V} \mid\end{array}$ & $\begin{array}{l}\overline{0} \\
\dot{0} \\
V_{1}\end{array}$ \\
\hline & 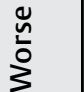 & 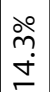 & $\mid \begin{array}{l}0 \\
0 \\
0 \\
0\end{array}$ & $\begin{array}{l}0 \\
0 \\
0 \\
0\end{array}$ & 实 & ठ̊ํ. & 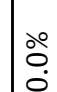 & $\frac{\stackrel{\circ}{\circ}}{\dot{\sigma}}$ & 官 & @ \\
\hline$\frac{\vec{\Phi}}{\Phi}$ & $\begin{array}{l}\stackrel{\mathscr{E}}{\tilde{N}} \\
\stackrel{n}{n}\end{array}$ & $\begin{array}{l}\stackrel{\circ}{\check{r}} \\
\end{array}$ & 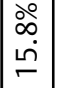 & $\begin{array}{l}\stackrel{\circ}{0} \\
\stackrel{\dot{d}}{ }\end{array}$ & $\begin{array}{l}\stackrel{\circ}{2} \\
\dot{\infty}\end{array}$ & $\begin{array}{l}\stackrel{\circ}{\tilde{\Xi}} \\
\stackrel{\sim}{2}\end{array}$ & $\begin{array}{l}\stackrel{\circ}{0} \\
\infty \\
\sim \\
N\end{array}$ & $\frac{\text { வீ}}{\check{\sigma}}$ & $\begin{array}{l}\stackrel{0}{0} \\
0 \\
0\end{array}$ & 总 \\
\hline 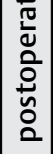 & 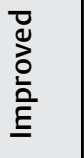 & $\begin{array}{l}\stackrel{\circ}{0} \\
\stackrel{0}{\wedge}\end{array}$ & 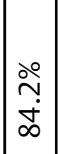 & $\begin{array}{l}\stackrel{\circ}{0} \\
\dot{0} \\
\infty\end{array}$ & $\begin{array}{l}\stackrel{2}{\hat{~}} \\
\bar{\sigma}\end{array}$ & $\begin{array}{l}\stackrel{2}{\hat{~}} \\
\dot{\infty} \\
\infty\end{array}$ & 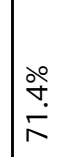 & \begin{tabular}{|l}
$\stackrel{0}{\infty}$ \\
$\dot{\infty}$ \\
\end{tabular} & $\begin{array}{l}\stackrel{0}{0} \\
\dot{0} \\
0\end{array}$ & ஹે \\
\hline $\mid \begin{array}{l}\tilde{E} \\
\mathbf{Z} \\
\mathrm{O} \\
\mathrm{E} \\
\mathrm{m}\end{array}$ & 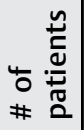 & \pm & $\stackrel{\rho}{\Gamma}$ & $\stackrel{\sim}{\sim}$ & $\simeq$ & $\wedge$ & 1 & $\mp$ & $\mathrm{m}$ & ñ \\
\hline & 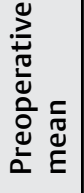 & 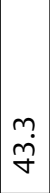 & $\overline{\vec{Y}}$ & $\begin{array}{l}0 \\
\dot{\gamma}\end{array}$ & $\underset{\sim}{\stackrel{\sim}{i}}$ & ָิ & $\mid \begin{array}{l}0 \\
\infty \\
m \\
m\end{array}$ & $\overline{\dot{q}}$ & $\frac{m}{6}$ & $\mid \begin{array}{l}\infty \\
\dot{\sigma} \\
\dot{q}\end{array}$ \\
\hline & ্ָত & ํ. & 高 & $\overline{\overline{2}}$ & $\stackrel{\sim}{\stackrel{N}{\sim}}$ & $\stackrel{m}{\stackrel{n}{N}}$ & 离 & 织 & $\frac{6}{2}$ & 胥 \\
\hline
\end{tabular}

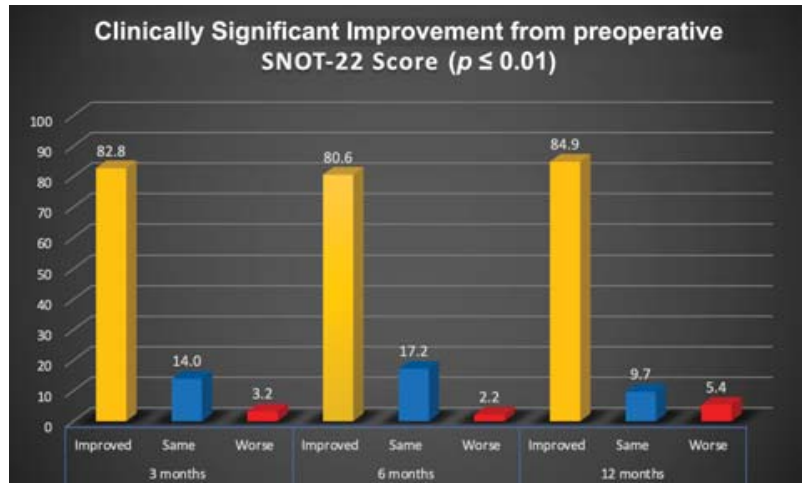

Fig. 1 Overall Group A Improvement Results (Percentage Change).

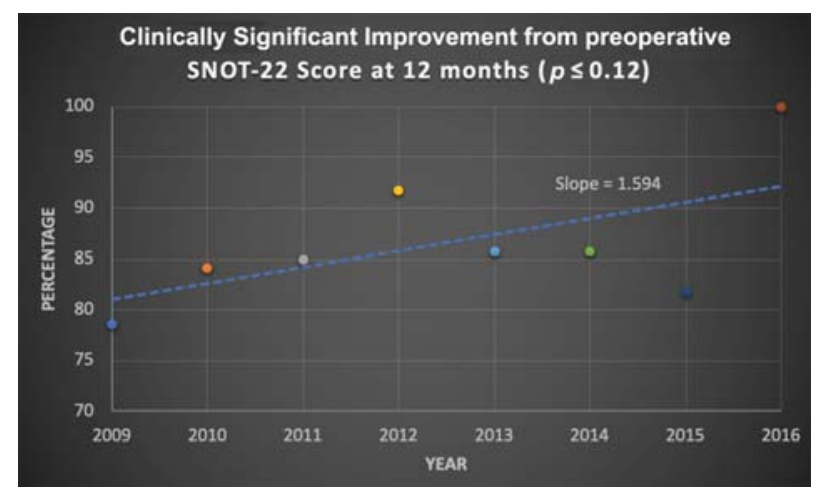

Fig. 2 Group A 8-year trend analysis of 12-month rates.

MSSC of 25.2. At 6 months, the MSSC was 26.2, with postoperative $78.9 \%$ improvement, $21.1 \%$ same, and $0 \%$ worsened compared with their initial SNOT-22 score. At 12 months, the MSSC was 21.7, with $84.2 \%$ improved, $10.5 \%$ the same, and $5.3 \%$ worsened compared with their preoperative SNOT-22 score.

In 2011, 20 patients in Group A had a preoperative mean of 43.6. At 3 months, the MSSC was 24.7 with reported scores of $80 \%$ improvement, $20 \%$ same, and $0 \%$ worsened from their pre-op SNOT-22 score. At 6 months, the MSSC was 24.4, with rates reported like at 3 months postoperatively, $80 \%$ improvement, $20 \%$ same, and $0 \%$ worsened. At 12 months, the MSSC was 25.0 , with $85 \%$ improvement, $10 \%$ same, and $5 \%$ worsened.

In 2012, 12 patients in Group A had a preoperative mean of 32.4. At 3 months, the MSSC was 20.9 with reported scores of $91.7 \%$ improvement, $8.3 \%$ the same, and $0 \%$ worsened. At 6 months, the MSSC was 18.1 with $75 \%$ improvement, $25 \%$ same, $0 \%$ worsened. At 12 months, the MSSC was 19.4 with $91.7 \%$ improvement, $0 \%$ the same, and $8.3 \%$ reported a score that was greater than their initial score. In this Group, 3 patients were indicated to be revision surgeries with a revision preoperative mean improvement of 31 . At 3 months, the MMSC was 1 , and $66.7 \%$ improved, $33.3 \%$ worsened. At 6 months, the MMSC was 11.3 with $66.7 \%$ improvement, $33.3 \%$ worse. At 12 months, the MMSC was 22 and $100 \%$ improved.

In 2013, 7 patients in Group A had a preoperative mean of 42.7. At 3 months postoperatively, the MSSC was 31.7, with a 
reported $85.7 \%$ improvement, $14.3 \%$ the same, and $0 \%$ worsened. At 6 months, the MSSC was 26.9 with similar results as reported during the 3 months follow-up (85.7\% improvement, $14.3 \%$ the same, and $0 \%$ worsened). At 12 months postoperatively, the MSSC was 25.9 with identical rates being reported as at 3 months and 6 months post-op (85.7\% improvement, $14.3 \%$ the same, and $0 \%$ worsened).

In 2014, 7 patients in Group A had a preoperative mean of 38.6. At 3 months, the MSSC was 26.4 with surgical success rate reported at $71.4 \%, 28.6 \%$ reported no change, and $0 \%$ reported worsened symptom scores. At 6 months the MSSC was 23.4, with $85.7 \%$ reported scores that improved, and $14.3 \%$ reported scores that had not changed significantly from their preoperative score. At 12 months postoperatively, the MSSC was 30.8 with the same rates being reported as at 6 months (85.7\% improved, $14.3 \%$ the same, and $0 \%$ worsened.)

In 2015, 11 patients in Group A had a preoperative mean of 43.1. At 3 months, the MSSC was 19.7 with $81.8 \%$ reporting scores that improved from their preoperative SNOT-22 score, 9.1\% reporting scores that did not change significantly, and $9.1 \%$ worsened from their preoperative SNOT-22 score. At 6 months postoperatively, the MSSC was 25.4, with rates being reported as $72.7 \%$ improved, $27.3 \%$ the same, and $0 \%$ worsened. At 12 months postoperatively, the MSSC was 25.2 with $81.8 \%$ significantly lower scores than their initial, $18.2 \%$ reporting scores that did not change significantly, and $0 \%$ reporting worsened scores.

In 2016, 3 patients in Group A had a preoperative mean of 61.3. At 3 months, the MSSC was 53.3 with $100 \%$ reporting improvement. At 6 months, the MSSC was 49.7, and at 12 months the MSSC was 30.3. All of the patients reported $100 \%$ at each postoperative period ( 3 months, 6 months, and 12 months).

From April 2009 to December 2016, 282 patients completed SNOT-22 questionnaires with at least 1 but not all of the postoperative questionnaires. Group B had a total number of 240 patients at 3 months, 180 at 6 months, and 121 at 12 months. A total of 48 patients (17.0\%) were lost to follow up at 3 months, 102 patients (36.2\%) at 6 months, and 161 patients $(57.1 \%)$ at 12 months.

The average loss over the 8-year period for Group B was: 3 months, 14.6\%; 6 months, 33.7\%; 12 months, 56.1\%

Group B results are broken down in -Table 2. Group B showed similar results when compared with Group A, regarding the surgical success rates at each postoperative period, $71.3 \%$, $78.3 \%$, and $84.3 \%$, respectively ( $\mathrm{p} \leq 0.01$ ) ( Fig. 3 ). At 3 months postoperatively, 240 patients in Group B on average reported $71.25 \%$ improvement, $22.5 \%$ the same, and $6.25 \%$ worsened from their preoperative score. At 6 months postoperatively, 180 patients in Group B on average reported 78.3\% improvement, $19.44 \%$ the same, $2.22 \%$ worsened from their preoperatively score. At 12 months postoperatively, 121 patients in Group B on average reported $84.3 \%$ improvement, $11.6 \%$ the same, and $4.1 \%$ worsened from their preoperative score.

In Group B, at 3 months, 9 out of the 240 surgeries were revisions ( 2 in 2011, 6 in 2012, and 1 in 2013). The patient rates of surgical efficacy with revision surgeries showed 44.4\% significant improvement, $33.3 \%$ did not report a significant change, and $22.2 \%$ reported worse scores. At

\begin{tabular}{|c|c|c|c|c|c|c|c|c|c|c|}
\hline \multirow{4}{*}{ 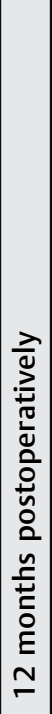 } & ڤั & $\begin{array}{l}\text { ㅇํ } \\
\text { ஸ̊ } \\
-\end{array}$ & $\stackrel{\circ \circ}{\text { ஸे }}$ & $\begin{array}{l}\stackrel{\circ}{\text { ஸे }} \\
\text { †े }\end{array}$ & $\begin{array}{l}\stackrel{\circ}{m} \\
\text { ம் }\end{array}$ & $\mid \begin{array}{l}\circ \\
\circ \\
0\end{array}$ & ○̊ํ. & $\begin{array}{l}\text { O̊ } \\
\text { Oे }\end{array}$ & \begin{tabular}{|l}
$\stackrel{\circ}{0}$ \\
Oे
\end{tabular} & $\frac{\stackrel{\circ}{\leftarrow}}{\dot{\gamma}}$ \\
\hline & $\frac{\mathscr{U}}{\mathrm{E}}$ & 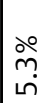 & $\frac{\stackrel{\circ}{\stackrel{\infty}{\infty}}}{\infty}$ & $\stackrel{\circ}{\circ}$ & 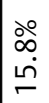 & $\mid \begin{array}{l}\circ 0 \\
m \\
\infty\end{array}$ & $\begin{array}{l}\stackrel{ }{\circ} \\
\stackrel{\infty}{\circ}\end{array}$ & $\begin{array}{l}\text { ○े } \\
\text { ஸे }\end{array}$ & $\begin{array}{l}\circ \\
\text { O̊ } \\
0\end{array}$ & 宅 \\
\hline & 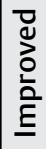 & $\begin{array}{l}\stackrel{\circ}{\circ} \\
\stackrel{\infty}{\infty}\end{array}$ & $\begin{array}{l}\text { 웅 } \\
\text { م. }\end{array}$ & 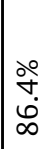 & $\begin{array}{l}\stackrel{\circ}{\circ} \\
\infty \\
\infty\end{array}$ & $\begin{array}{l}\stackrel{0}{ヘ} \\
\bar{\sigma} \\
\end{array}$ & $\begin{array}{l}\stackrel{0}{\infty} \\
\infty \\
\dot{\infty}\end{array}$ & $\begin{array}{l}\text { ○̊ } \\
\text { ஸे }\end{array}$ & 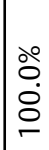 & $\begin{array}{l}\stackrel{\circ}{m} \\
\dot{\infty} \\
\dot{\infty}\end{array}$ \\
\hline & 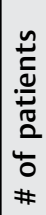 & 9 & $\stackrel{m}{\sim}$ & $\approx$ & $\stackrel{\sigma}{-}$ & $\simeq$ & $\mp$ & $\simeq$ & $m$ & $\stackrel{\nwarrow}{\simeq}$ \\
\hline \multirow{4}{*}{ 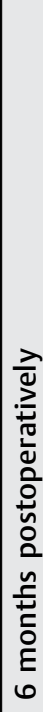 } & ڤั & ঐ̊ & $\begin{array}{l}\text { O̊ } \\
\text { Oீ. }\end{array}$ & $\begin{array}{l}\text { 우 } \\
\text { ơ }\end{array}$ & \begin{tabular}{|l} 
ㅇํ \\
ナர
\end{tabular} & $\begin{array}{l}\circ \\
0 \\
0\end{array}$ & $\frac{\stackrel{0}{\check{C}}}{\check{\sigma}}$ & $\begin{array}{l}\text { O̊ } \\
\text { Oீ. }\end{array}$ & i̊n & $\stackrel{\stackrel{\circ}{N}}{\mathrm{~N}}$ \\
\hline & $\stackrel{\mathscr{E}}{\frac{E}{n}}$ & 总 & $\begin{array}{l}\stackrel{\circ}{6} \\
\stackrel{N}{N}\end{array}$ & $\begin{array}{l}\circ 0 \\
\infty \\
\stackrel{1}{\sim}\end{array}$ & $\begin{array}{l}\stackrel{\partial}{6} \\
\dot{v} \\
\dot{r}\end{array}$ & $\begin{array}{l}o \circ \\
\stackrel{\circ}{\circ} \\
\stackrel{1}{\square}\end{array}$ & $\frac{\stackrel{\circ}{\check{\sigma}}}{\sigma}$ & $\begin{array}{l}\stackrel{\circ}{人} \\
\stackrel{\text { N }}{\text { N }}\end{array}$ & $\begin{array}{l}\circ 0 \\
0 \\
0\end{array}$ & $\begin{array}{l}\stackrel{\circ}{+} \\
\stackrel{+}{\leftarrow}\end{array}$ \\
\hline & 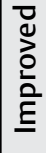 & 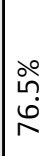 & ㅇํㅁ & $\begin{array}{l}\stackrel{\circ}{\sim} \\
\stackrel{+}{ர}\end{array}$ & \begin{tabular}{|l}
$\stackrel{0}{ }$ \\
$\infty$ \\
$\grave{\infty}$
\end{tabular} & $\begin{array}{l}\stackrel{\circ}{\circ} \\
\dot{\infty} \\
\end{array}$ & $\begin{array}{l}\circ 0 \\
\infty \\
\bar{\infty} \\
\infty\end{array}$ & $\stackrel{\circ}{\stackrel{\circ}{\aleph}}$ & $\begin{array}{l}\text { ํㅜ } \\
\text { ஸे } \\
\text { ஸे }\end{array}$ & $\begin{array}{l}\stackrel{\circ}{0} \\
\stackrel{\infty}{\infty} \\
\infty\end{array}$ \\
\hline & 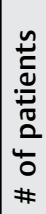 & mे & $\bar{m}$ & $\bar{m}$ & $\approx$ & $\bar{\sim}$ & $\mp$ & $\approx$ & $\infty$ & $\infty$ \\
\hline \multirow{4}{*}{ 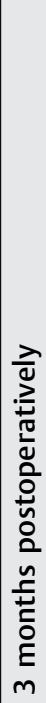 } & 岕 & $\stackrel{\stackrel{\circ}{\dddot{m}}}{\stackrel{+}{+}}$ & $\begin{array}{l}\text { ○̊ } \\
\text { ஸे }\end{array}$ & \begin{tabular}{|l} 
O̊ \\
Oे
\end{tabular} & $\begin{array}{l}\text { ○̊ } \\
\text { mे }\end{array}$ & $\begin{array}{l}\stackrel{\circ}{\checkmark} \\
\sim \\
\sim\end{array}$ & 永 & $\stackrel{\text { mे }}{m}$ & 吕 & $\begin{array}{l}\stackrel{\circ}{\text { mे }} \\
\text { (n) }\end{array}$ \\
\hline & 亗 & $\frac{\stackrel{\partial}{\sim}}{\grave{\sim}}$ & $\begin{array}{l}\stackrel{\circ}{0} \\
\stackrel{1}{n}\end{array}$ & $\begin{array}{l}\stackrel{\circ}{0} \\
\stackrel{\sim}{N}\end{array}$ & $\begin{array}{l}\stackrel{\circ}{m} \\
\dot{\forall}\end{array}$ & $\begin{array}{l}\text { م̊ } \\
\text { ஸ் }\end{array}$ & 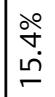 & 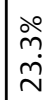 & ○̊ & ํํำ \\
\hline & 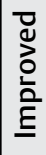 & $\begin{array}{l}\stackrel{\circ}{~} \\
\stackrel{\sigma}{\sigma}\end{array}$ & 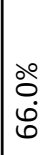 & 월 & $\begin{array}{l}\stackrel{\circ}{\check{\Gamma}} \\
\stackrel{\infty}{\infty}\end{array}$ & $\mid \begin{array}{l}\stackrel{0}{N} \\
\dot{b} \\
0\end{array}$ & $\begin{array}{l}\text { 亏े } \\
\text { ம் }\end{array}$ & $\begin{array}{l}\stackrel{\circ}{m} \\
\stackrel{n}{r}\end{array}$ & $\begin{array}{l}\stackrel{0}{\sigma} \\
\infty \\
\infty\end{array}$ & m̊ \\
\hline & 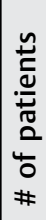 & $\mathscr{F}$ & 웃 & $\bar{\gamma}$ & $\stackrel{\infty}{\sim}$ & $\lesssim$ & $r$ & $\stackrel{\text { m }}{ }$ & $a$ & $\stackrel{\nabla}{N}$ \\
\hline & 竎 & 융 & 을 & 듕 & $\frac{N}{\stackrel{N}{N}}$ & $\frac{m}{\delta}$ & 市 & ำ & $\frac{0}{\varnothing}$ & 定 \\
\hline
\end{tabular}




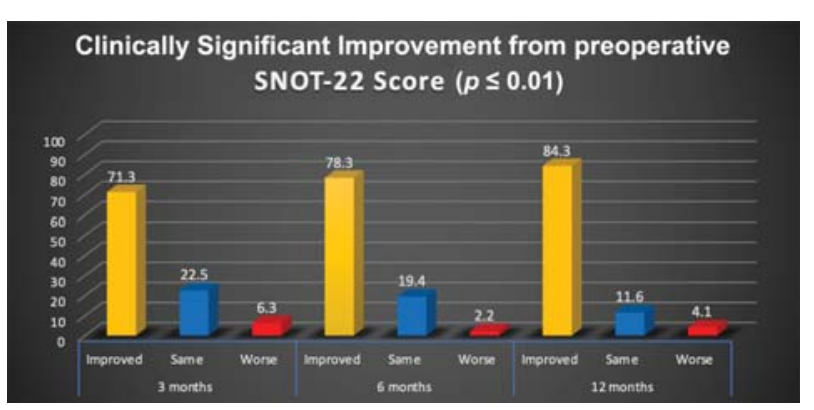

Fig. 3 Overall Group B Improvement Results (Percentage Change).

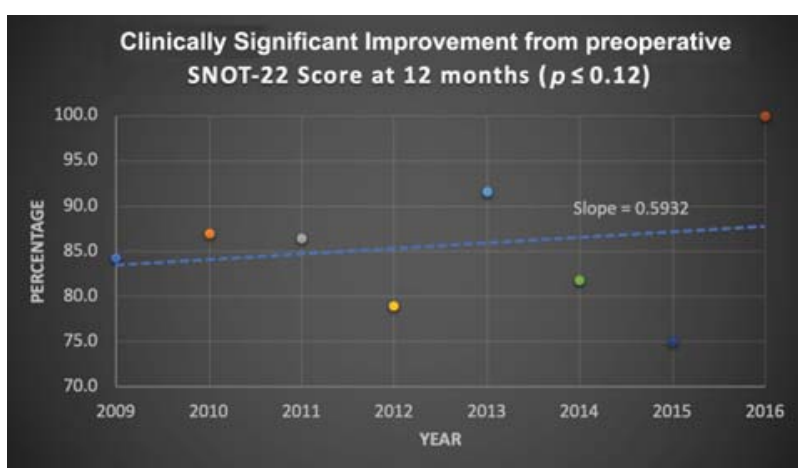

Fig. 4 Group B 8-year trend analysis of 12-month rates.

6 months, 5 out of the 180 surgeries were revisions ( 1 in 2009, 1 in 2011, and 3 in 2012) and illustrated 60\% improvement, $20 \%$ the same, and $20 \%$ worsened. At 12 months postoperatively, 3 out of the 121 surgeries (all 3 in 2012) were revisions and $100 \%$ had an improved SNOT-22 score. An 8 -year trend analysis of year-to-year surgical success rates at 12 months postoperatively for Group B (-Fig. 4) illustrated an improvement trend over time with an ANOVA linear rate of $0.593(p \leq 0.66)$.

Finally, the mean point change over time (-Fig. 5), from all patients who completed the preoperative and at least one postoperative questionnaire, has shown that patients experienced statistically significant change from the preoperative at 3 months $(p<0.0001)$ and at 6 months $(p<0.0001)$. Then, at 6 months, SNOT-22 scores plateaued and between 6 months and 12 months postoperatively, patients did not report a statistically significant change in SNOT-22 scores $(p<0.73)$.

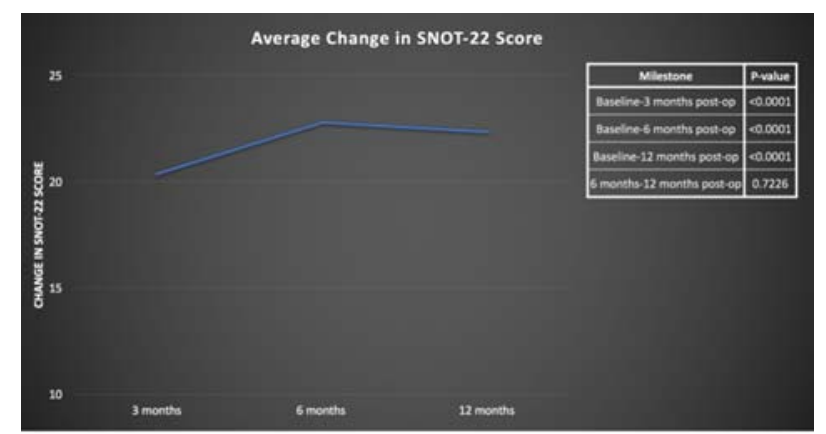

Fig. 5 Mean Point Change in SNOT-22 Scores (2009-2016).

\section{Discussion}

The present study is important because it observes the longterm efficacy of sinus surgery and determined an optimal time for evaluation for surgical efficacy. In addition, the study also observed if the efficacy of sinus surgery improved with time with experience.

These results analyzed the patient population at a tertiary medical center undergoing sinus surgery over the years and their long-term rates of surgical efficacy using their SNOT-22 scores. Evaluation of SNOT-22 scores helped to compare each year and observe a general trend of improvement in the rates of surgical efficacy over time through a multisurgeon center. At 12 months postoperatively, when comparing the surgical success rates for each of the years, a positive trend was observed over time. The surgical success rates for both groups, A and B, were found to be similar. After observing the trends both in Group A and B, we observed that, in general, patients undergoing sinus surgery at a tertiary medical center showed $84.9 \%$ improvement in sinus disease symptoms by 12 months postoperatively. Other factors have contributed to the $15.1 \%$ variable.

Research has shown that the presence of polyps in patients with CRS has been highly correlated with a negative impact on the QOL scores, ${ }^{10}$ as well as with a higher need for revision surgery and less improvement than patients with CRS without polyps. ${ }^{11}$ In addition, it is not clear whether or not smoking has a direct effect on postoperative SNOT-22 scores. White et al suggest that there is no difference in QOL scores in smokers and nonsmokers after sinus surgery. ${ }^{12}$ Alternatively, some studies suggest that while both smokers and nonsmokers may improve significantly from their preoperative QOL scores, some smokers experienced worsening in postoperative QOL scores after 6 months, depending on the smoking volume, ${ }^{13}$ and had a higher rate of revision surgery $^{14}$ compared with nonsmokers, $20 \%$ versus $7 \%$, respectively.

Despite research showing different prognostic factors for patients with different indications of surgery, all of the surgical candidates were asked to fill out a SNOT-22 form preoperatively. Furthermore, patients were grouped into two groups that depended solely on full completion of the SNOT-22 forms at each milestone. Patients who skipped a question on the survey were also excluded from both of the groups because they were artificially lowering their score. Although statistical bias is prevented by inclusion and exclusion criteria, the classifications of surgical indications may have swayed the statistical results.

In addition, a possible response bias could be attributed due to the fact that all of the values for SNOT-22 were selfreported and that the volume of patients lost to follow-up may pose a shift in the outcome. However, similar results were obtained in group A and group B, verifying that, even with more lenient inclusion criteria, similar results were obtained.

Furthermore, the study examined the whole patient population without considering demographics, the indication for surgery, past health history, smoking, or any use of 
long-term medications. Future studies could include creating sub-cohorts dependent on the different factors, such as the indication for surgery and demographics to help better understand the rates of surgical efficacy under each subdivision, but doing so will yield toward statistical bias, unlike when looking at the population as a whole.

\section{Conclusion}

Patients $\geq 18$ years old undergoing sinus surgery at a tertiary medical center showed an $84.9 \%$ improvement by 12 months postoperatively. Long-term analysis of surgical efficacy over the years and mean point change in SNOT-22 showed no difference between 6 months postoperatively and 12 months, signifying 6 months as an effective evaluation for surgical efficacy. The year to year 8-year trend at 12-months postoperatively shows an upward trend toward improvement in surgical success over the years but not to the level of statistical significance.

The rates of surgical success at 3 months and 6 months postoperatively averaged to $84.2 \%$ and $82.96 \%$ respectively, with a $95 \%$ confidence interval. The 8 -year trend analysis of year-to-year surgical success highlights 3-month and 6month postoperative data as not being statistically significant ( $p \leq 0.39$ and $p \leq 0.42$, respectively).

After 12 months postoperatively, an overall average of $84.9 \%$ of patients $(p \leq 0.01)$ that were analyzed in the present prospective study, from both Group A and Group B, reported an improvement in QOL. Similar rates were also obtained at 6 months with $80.6 \%$ within Group A versus $78.3 \%$ within Group B.

Throughout each of the different postoperative periods, despite the inclusion criteria for each of the groups, we found that Group A and Group B had similar results at each of the designated postoperative milestones. When comparing surgical success rates on a year-to-year basis for Group A and Group B, we found that patients who completed all SNOT-22 forms had statistically better results on a year-to-year basis than patients who did not complete all SNOT-22 forms.

\section{Conflicts of Interests}

The authors have no conflicts of interests to declare.

\section{Acknowledgments}

Cedars Sinai Sinus Center: David M. Alessi, MD; Babak Azizzadeh, MD; Gary S. Bellack, MD; Henry Chen, MD; Matthew L. Finerman, MD; Garrett Herzon, MD; Babak Larian, MD; Gene C. Liu, MD; Christina McAlpin, MD; Anita N. Newman, MD; Madison F. Richardson, MD; Robert O. Ruder, MD; Daryoush Saadat, MD; Nicholas L.
Schenck, MD; Raj P. TerKonda, MD; Arthur Wu, MD; Mani H. Zadeh, MD; Irina Shemetova, MBA; Sonam Kapadia, MHA; Stephanie Hopp, MS, MHS.

\section{References}

1 Hirsch AG, Stewart WF, Sundaresan AS, et al. Nasal and sinus symptoms and chronic rhinosinusitis in a population-based sample. Allergy 2017;72(02):274-281. Doi: 10.1111/all.13042

2 Rudmik L. Chronic rhinosinusitis: an under-researched epidemic. J Otolaryngol Head Neck Surg 2015;44(01):11. Doi: 10.1186/ s40463-015-0064-8

3 Tint D, Kubala S, Toskala E. Risk factors and Comorbidities in chronic Rhinosinusitis. Curr Allergy Asthma Rep 2016;16(02):16

4 Macdonald KI, McNally JD, Massoud E. The health and resource utilization of Canadians with chronic rhinosinusitis. Laryngoscope 2009;119(01):184-189. Doi: 10.1002/lary.20034

5 Goetzel RZ, Long SR, Ozminkowski RJ, Hawkins K, Wang S, Lynch W. Health, absence, disability, and presenteeism cost estimates of certain physical and mental health conditions affecting U.S. employers. J Occup Environ Med 2004;46(04):398-412. Doi: 10.1097/01.jom.0000121151.40413.bd

6 Gliklich RE, Metson R. The health impact of chronic sinusitis in patients seeking otolaryngologic care. Otolaryngol Head Neck Surg 1995;113(01):104-109. Doi: 10.1016/s0194-5998(95) 70152-4

7 Caulley L, Thavorn K, Rudmik L, Cameron C, Kilty SJ. Direct costs of adult chronic rhinosinusitis by using 4 methods of estimation: Results of the US Medical Expenditure Panel Survey. J Allergy Clin Immunol 2015;136(06):1517-1522. Doi: 10.1016/j.jaci.2015. 08.037

8 Rudmik L, Soler ZM, Mace JC, DeConde AS, Schlosser RJ, Smith TL. Using preoperative SNOT-22 score to inform patient decision for Endoscopic sinus surgery. Laryngoscope 2015;125(07): 1517-1522. Doi: 10.1002/lary.25108

9 Hopkins C, Slack R, Lund V, Brown P, Copley L, Browne J. (2009), Long-term outcomes from the English national comparative audit of surgery for nasal polyposis and chronic rhinosinusitis. The Laryngoscope 119:2459-2465. doi:10.1002/lary.20653

10 Abdalla S, Alreefy H, Hopkins C. Prevalence of sinonasal outcome test (SNOT-22) symptoms in patients undergoing surgery for chronic rhinosinusitis in the England and Wales National prospective audit. Clin Otolaryngol 2012;37(04):276-282. Doi: 10.1111/j.1749-4486.2012.02527.x

11 Deal RT, Kountakis SE. Significance of nasal polyps in chronic rhinosinusitis: symptoms and surgical outcomes. Laryngoscope 2004;114(11):1932-1935. Doi: 10.1097/01.mlg.0000147922. 12228.1f

12 White LC, Kazi AA, Jang DW, Gurrola J, Kountakis SE. The effect of smoking on quality of life following sinus surgery: 10-year followup. ORL J Otorhinolaryngol Relat Spec 2015;77(01):39-43

13 Rudmik L, Mace JC, Smith TL. Smoking and endoscopic sinus surgery: does smoking volume contribute to clinical outcome. Int Forum Allergy Rhinol 2011;1(03):145-152. Doi: 10.1002/ alr.20045

14 Krzeski A, Galewicz A, Chmielewski R, Kisiel M. Influence of cigarette smoking on endoscopic sinus surgery long-term outcomes. Rhinology 2011;49(05):577-582 